\title{
A Factor Graph-Based Iterative Detection of Faster-than-Nyquist Signaling in the Presence of Phase Noise and Carrier Frequency Offset
}

\author{
Xiaotong Qi, Nan Wu*, Hua Wang, Weijie Yuan \\ School of Information and Electronics, Beijing Institute of Technology, Beijing, China
}

\section{Abstract}

With the increasing demand for higher spectral efficiency in wireless communications, faster-than-Nyquist (FTN) signaling has been rediscovered to increase transmission rate without expanding signaling bandwidth. Most existing studies focus on low-complexity FTN receiver design by assuming perfect synchronization. In practice, however, phase noise (PHN) and carrier frequency offset (CFO) may degrade the performance of FTN detector significantly. In this paper, we develop iterative FTN detector in the presence of PHN and CFO in a factor graph framework. Wiener process is employed to model the time evolution of nonstationary channel phase. The colored noise imposed by sampling of FTN signaling is approximated by autoregressive model. Based on the factor graph constructed, messages are derived on the two subgraphs, i.e., PHN and CFO estimation subgraph and the FTN symbol detection subgraph. We propose two methods to update the messages between subgraphs, namely, Gaussian approximation via Kullback-

\footnotetext{
${ }^{*}$ Corresponding author

Email address: \{xtseven, wunan, wanghua, wjyuan\}@bit.edu.cn (Xiaotong Qi, Nan $\mathrm{Wu}^{*}$, Hua Wang, Weijie Yuan)

Preprint submitted to Digital Signal Processing

December 18, 2016
}

(C) 2016. This manuscript version is made available under the Elsevier user license http://www.elsevier.com/open-access/userlicense/1.0/ 
Leibler divergence (KLD) minimization and the combined sum-product and variational message passing (SP-VMP), both of which enable low-complexity parametric message passing. The proposed SP-VMP algorithm can provide closed-form expressions for parameters updating. Moreover, conjugate gradient (CG) method is adopted to solve the maximum a posteriori probability (MAP) estimation of CFO with fast convergence speed. Simulation results show the superior performance of the proposed algorithm compared with the existing methods and verify the advantage of FTN signaling compared with the Nyquist counterpart.

Keywords: Faster-than-Nyquist signaling, phase noise (PHN), carrier frequency offset (CFO), factor graphs, colored noise, sum-product algorithm (SPA), variational message passing (VMP)

\section{Introduction}

Faster-than-Nyquist (FTN) signaling, first proposed by Mazo in 1970s [1], has been rediscovered in recent years as a promising technique to improve spectral efficiency while preserving the signaling bandwidth [2-4]. It has been proved that FTN signaling can attain the same asymptotic error probability as the Nyquist signaling as long as the packing factor is above the Mazo limit [5]. Nevertheless, since the shaping pulses of FTN signaling are packed to transmit more symbols during the same time interval $T$, the intersymbol interference (ISI) is unavoidable.

Many studies have been performed to eliminate the intentional ISI imposed by FTN [6-11]. In [6], the FTN signaling is considered as a convolutional code and Viterbi algorithm is applied for symbol detection. A 
maximum a posteriori probability (MAP) detector with successive interference cancelation (SIC) is employed in [7]. In [8], a reduced-complexity turbo equalization-based M-algorithm BCJR (M-BCJR) algorithms have been proposed. A frequency-domain equalization (FDE)-based FTN receiver is studied in [9], where the impact of ISI of FTN signaling is approximated by circulant matrix structure, which enables low-complexity minimum mean squared error (MMSE) symbol detection. The FDE-aided iterative detector is employed in a three-stage-concatenated FTN system in [10] to further improve the performance. However, the colored noise imposed by sampling the FTN signaling is not fully considered in $[9,10]$. Using autoregressive (AR) process to model the colored noise, a graph-based linear MMSE (LMMSE) equalizer is developed in [11].

The existing studies on receiver design of FTN signaling assume perfect synchronization. In practice, however, local oscillator instabilities of both transmitter and receiver will lead to phase noise (PHN), which becomes one of the major impairments to the performance of communication systems. It is further exacerbated by the recent demand for larger bandwidth at higher frequencies, where PHN becomes much more severe as the oscillator frequency increases. There have been many researches to address the impact of PHN for Nyquist signaling [12-17]. In [14] an iterative decoding algorithm for channels impacted by strong PHN is proposed, which utilizes the sumproduct algorithm (SPA) [18] to derive the MAP symbol detector. Canonical distribution approach [19] has been employed to represent the probability density function (pdf) of PHN by Tikhonov distribution. In [15], variational bounding is employed to design the iterative detection algorithm, and then 
Taylor-series expansion is used to linearize the system model and extended Kalman smoothing is applied to calculate the soft estimation of PHN. Based on variational inference, [16] constraints the free distribution as Gaussian or Dirac delta function to further reduce the complexity. In [17], by partitioning the received frame into partial blocks, the PHN is estimated based on discrete cosine transform (DCT) expansion. The estimation accuracy of the above algorithms degrade rapidly in the presence of carrier frequency offset (CFO). Although many pilot-aided CFO estimators can be employed [20], long training sequence leads to spectral efficiency loss. On the other hand, the presence of strong PHN may also degrade the accuracy of CFO estimation. Therefore, joint estimation of CFO and PHN should be considered. In [21], a joint detection and decoding algorithm in the presence of PHN and $\mathrm{CFO}$ is proposed based on factor graph, where $\mathrm{CFO}$ is quantized with equally spaced level and processed in parallel for each quantization value.

In this paper, we develop an iterative detection algorithm for FTN signaling in the presence of PHN and CFO. The impact of PHN and CFO is modeled as Wiener process and the colored noise imposed by the sampling of FTN signaling is approximated by AR model. Building on this, the joint posterior distribution is factorized efficiently and the corresponding factor graph is constructed. For ease of exposition, we further divide the whole factor graph into two subgraphs, namely, PHN and CFO estimation and FTN symbol detection, and the updating rules of messages are derived on each subgraphs. The proposed algorithm results in low-complexity parametric message passing to detect FTN symbols iteratively on factor graph. Simulations results demonstrate the superior performance of the proposed algorithm 
in FTN system compared with the state-of-the-art methods and the Nyquist counterpart. The main contributions of this paper are summarized in the following:

- Different from existing works that assume perfect synchronization, we study more practical situation of FTN signaling detection in the presence of PHN and CFO. Based on the efficient factorization of the joint posterior distribution, we solve the joint detection and estimation problem in factor graph framework.

- We propose two methods to update the messages between subgraphs, i.e., Gaussian approximation via Kullback-Leibler divergence (KLD) minimization and the combined sum-product and variational message passing (SP-VMP). Both of the two methods enable us to perform parametric message passing on factor graph. Especially, the proposed SP-VMP algorithm results in closed-form parameters updating rules.

- Instead of performing quantization on CFO as in [21], we use Dirac delta function with the MAP estimate to represent the message from the variable node of $\mathrm{CFO}$, which avoids the complex parallel processing for each quantized $\mathrm{CFO}$ value in [21]. Conjugate gradient (CG) method is adopted to solve the optimization with faster convergence speed.

The remainder of this paper is organized as follows. Section II describes the system model. In Section III, the probabilistic model of FTN signaling detection is presented and the factor graph is constructed. The updating rules of messages are derived on factor graph. The simulation results and 
discussions are given in Section IV. Finally, conclusions are drawn in Section VI.

Notations: We use boldface capital letter to denote a matrix while boldface lower-case letter for a vector. $\Re(\cdot),(\cdot)^{*}$ represent the real part, complex conjugate of a complex number; $(\cdot)^{T},(\cdot)^{H}$ and $(\cdot)^{-1}$ are the transpose, conjugate transpose and the inverse operator, respectively; $g\left(m_{x}, v_{x} ; x\right)$ and $g_{\mathcal{C}}\left(m_{x}, v_{x} ; x\right)$ are real and complex Gaussian distribution of variable $x$ with mean $m_{x}$ and variance $v_{x}$, respectively; $\propto$ represents equality up to a constant normalization factor; $\mathbf{W}$ is the weight matrix; $\mathcal{S} \backslash s$ denotes all elements in the set $\mathcal{S}$ but $s$.

\section{System Model}

We consider a low-density parity-check (LDPC)-coded linearly modulated FTN system illustrated in Fig. 1. The mapping function from information

bits $\mathbf{d}=\left[d_{0}, \ldots, d_{N-1}\right]^{T}$ to coded symbols $\mathbf{x}=\left[x_{1}, \ldots, x_{K}\right]^{T}$ is denoted by $\mathbf{x}=\zeta_{\mathcal{C}}(\mathbf{d})$. The symbol sequence $\mathbf{x}$ is passed through a $T$-orthogonal rootraised-cosine (rRC) pulse shaping filter $g(t)$ with signaling rate $\frac{1}{\tau T}$, where $0<\tau \leq 1$ is the packing factor of FTN signaling and $T$ is the symbol interval of the Nyquist signaling. Then, the signal is transmitted over an additive white Gaussian noise (AWGN) channel. At receiver side, the signal is affected by PHN and CFO, which is given by

$$
s(t)=\left[\sum_{m} x_{m} g(t-n \tau T)+n(t)\right] e^{j \theta(t)},
$$

where $\theta(t)$ represents the time varying channel phase and $n(t)$ is an additive white circularly symmetric complex Gaussian noise with power spectral 
density $N_{0}$. Assuming perfect symbol timing between the transmitter and receiver, the received signal is passed though the matched filter and sampled with period $\tau T$. The signal sample at the $k$ th time instance is

$$
r_{k}=\int_{-\infty}^{+\infty} s(t) g^{*}(t-k \tau T) d t .
$$

Substituting (1) into (2) yields

$$
r_{k}=c_{k} e^{j \theta_{k}}+\omega_{k},
$$

where $c_{k}=\sum_{m=-\infty}^{\infty} x_{m} h_{m-k}$ represents the symbols affected by the FTN imposed ISI with $h_{m-k}=\int_{-\infty}^{+\infty} g(t-m \tau T) g^{*}(t-k \tau T) d t, \omega_{k}$ is the colored noise samples with autocorrelation function given as [22]

$$
R(m-k)=\mathbb{E}\left[\omega_{m} \omega_{k}^{*}\right]=N_{0} h_{m-k}
$$

We can use autoregressive model of the first order to approximate the colored noise process, which is expressed as

$$
\omega_{k}=\alpha \omega_{k-1}+\epsilon_{k}
$$

where $p\left(\epsilon_{k}\right)=g_{\mathcal{C}}\left(0, \sigma_{\epsilon}^{2} ; \epsilon_{k}\right)$, the parameters $\alpha$ and $\sigma_{\epsilon}^{2}$ can be obtained via Yule-Walker equation [23].

The time evolution of the channel phase due to PHN and $\mathrm{CFO}$ can be modeled by Wiener process. The discrete-time sample of $\theta(t)$ follows

$$
\theta_{k}=\theta_{k-1}+\varphi+\varepsilon_{k}
$$

where $\left\{\varepsilon_{k}\right\}$ is a real independent and identically distributed (i.i.d.) Gaussian sequence with $p\left(\varepsilon_{k}\right)=g\left(0, \sigma_{\varepsilon}^{2} ; \varepsilon_{k}\right), \varphi \triangleq 2 \pi \Delta f \tau T$ with frequency offset $\Delta f$ being a constant. ${ }^{1}$

\footnotetext{
${ }^{1} \Delta f$ is uniformly distributed in $\left[-f_{0}, f_{0}\right]$ and $\Delta f \tau T<1 / 2$.
} 
Although the length of ISI imposed by FTN signaling is infinite, due to the fast decay of the rRC pulse, we can approximate it by only considering length $L$. Using the notations $\mathbf{s}_{k} \triangleq\left[x_{k-L+1}, \ldots, x_{k}\right]^{T}$ and $\mathbf{h} \triangleq\left[h_{-(L-1)}, \ldots, h_{0}\right]^{T}$, we have $c_{k}=\mathbf{h}^{T} \mathbf{s}_{k}$. The state $\mathbf{s}_{k}$ can be represented by the following linear state-space model

$$
\mathbf{s}_{k}=\boldsymbol{\Lambda} \mathbf{s}_{k-1}+\mathbf{a} x_{k}
$$

where $\mathbf{a}=\left[\begin{array}{ll}\mathbf{0}^{T} & 1\end{array}\right]^{T}$ and $\boldsymbol{\Lambda}$ is a $L \times L$ matrix as

$$
\boldsymbol{\Lambda}=\left[\begin{array}{cc}
\mathbf{0} & \mathbf{I}_{L-1} \\
0 & \mathbf{0}^{T}
\end{array}\right]
$$

\section{Iterative Receiver for FTN Signaling Based on Bayesian Graph- ical Model}

Define $\mathbf{r} \triangleq\left[r_{0}, \ldots, r_{K-1}\right]^{T}, \boldsymbol{\theta} \triangleq\left[\theta_{0}, \ldots, \theta_{K-1}\right]^{T}, \boldsymbol{\omega} \triangleq\left[\omega_{0}, \ldots, \omega_{K-1}\right]^{T}$. The optimal MAP detection of $d_{k}$ can be obtained by maximizing the marginal $a$ posteriori probability $(\mathrm{APP}) p\left(d_{k} \mid \mathbf{r}\right)$, i.e.,

$$
\begin{aligned}
\hat{d}_{k} & =\underset{d_{k}}{\operatorname{argmax}} p\left(d_{k} \mid \mathbf{r}\right) \\
& =\underset{d_{k}}{\operatorname{argmax}} \sum_{\mathbf{d} \backslash d_{k}} \int p(\mathbf{d}, \boldsymbol{\theta}, \boldsymbol{\omega}, \varphi \mid \mathbf{r}) \mathrm{d} \boldsymbol{\theta} \mathrm{d} \boldsymbol{\omega} d \varphi .
\end{aligned}
$$

However, the marginalization in (9) is quite complicated due to the integration of the joint posterior distribution $p(\mathbf{d}, \boldsymbol{\theta}, \boldsymbol{\omega}, \varphi \mid \mathbf{r})$ with respect to large number of nuisance parameters. We propose to solve this problem through low-complexity message passing algorithm on factor graph. 
Based on Bayesian rules and the models in (5) and (6), the joint posterior distribution $p(\mathbf{d}, \boldsymbol{\theta}, \boldsymbol{\omega}, \varphi \mid \mathbf{r})$ can be factorized as

$$
\begin{aligned}
p(\boldsymbol{d}, \boldsymbol{\theta}, \boldsymbol{\omega}, \varphi \mid \boldsymbol{r}) \propto & \delta\left[\boldsymbol{x}-\zeta_{\mathcal{C}}(\boldsymbol{d})\right] p\left(\theta_{0}\right) p\left(\omega_{0}\right) p(\varphi) \\
& \times \prod_{k=1}^{K-1} \delta\left[c_{k}-\boldsymbol{h}^{T} \boldsymbol{s}_{k}\right] f_{\boldsymbol{s}_{k}}\left(\boldsymbol{s}_{k}, \boldsymbol{s}_{k-1}, x_{k}\right) p\left(r_{k} \mid c_{k}, \theta_{k}, \omega_{k}\right) \\
& \times p\left(\theta_{k} \mid \theta_{k-1}, \varphi\right) p\left(\omega_{k} \mid \omega_{k-1}\right)
\end{aligned}
$$

where $f_{\mathbf{s}_{k}}\left(\mathbf{s}_{k}, \mathbf{s}_{k-1}, x_{k}\right)$ represents the linear state-space model in (7). The variables $d_{k}, c_{k}, s_{k}, x_{k}$ are related by the constraint $f_{\mathbf{s}_{k}}\left(\mathbf{s}_{k}, \mathbf{s}_{k-1}, x_{k}\right), \delta\left[c_{k}-\boldsymbol{h}^{T} \boldsymbol{s}_{k}\right]$ and $\delta\left[\boldsymbol{x}-\zeta_{\mathcal{C}}(\boldsymbol{d})\right]$. The factor graph representing the factorization of (10) is illustrated in Fig. 3. Message passing algorithm, e.g, SPA, can be employed on the factor graph to calculate the marginal distribution efficiently [24]. In the following, we will derive the expressions of messages on factor graph. Specifically, we focus on two subgraphs, namely, PHN and CFO estimation and FTN symbol detection.

\subsection{Messages on the Subgraph of PHN and CFO Estimation}

The message from $p\left(r_{k} \mid c_{k}, \theta_{k}, \omega_{k}\right)$ to $\theta_{k}$ is denoted as $p_{d}\left(\theta_{k}\right)$, which can be calculated based on SPA rules as

$$
p_{d}\left(\theta_{k}\right)=\iint p_{d}\left(c_{k}\right) p_{d}\left(\omega_{k}\right) p\left(r_{k} \mid c_{k}, \theta_{k}, \omega_{k}\right) \mathrm{d} \omega_{k} \mathrm{~d} c_{k}
$$

We assume that $p_{d}\left(c_{k}\right)$ and $p_{d}\left(\omega_{k}\right)$ are in Gaussian form ${ }^{2}$, i.e., $p_{d}\left(c_{k}\right) \propto$ $g_{\mathcal{C}}\left(m_{c_{k}}^{\downarrow}, v_{c_{k}}^{\downarrow} ; c_{k}\right)$ and $p_{d}\left(\omega_{k}\right) \propto g_{\mathcal{C}}\left(m_{\omega_{k}}^{\downarrow}, v_{\omega_{k}}^{\downarrow} ; \omega_{k}\right)$. Then, (11) can be written

\footnotetext{
${ }^{2}$ By Gaussian approximation of data symbols, it can be verified that $p_{d}\left(c_{k}\right)$ and $p_{d}\left(\omega_{k}\right)$ are Gaussian distributions, which enables efficient parametric message passing.
} 
as

$$
\begin{aligned}
p_{d}\left(\theta_{k}\right) & \propto \int p_{d}\left(c_{k}\right) \exp \left\{-\frac{\left|r_{k}-m_{\omega_{k}}^{\downarrow}-c_{k} e^{j \theta_{k}}\right|^{2}}{2 v_{\omega_{k}}^{\downarrow}}\right\} \mathrm{d} c_{k} \\
& \propto \int \exp \left\{-\frac{\left|\left(r_{k}-m_{\omega_{k}}^{\downarrow}\right) e^{-j \theta_{k}}-c_{k}\right|^{2}}{2 v_{\omega_{k}}^{\downarrow}}\right\} \exp \left\{-\frac{\left|c_{k}-m_{c_{k}}^{\downarrow}\right|^{2}}{2 v_{c_{k}}^{\downarrow}}\right\} \mathrm{d} c_{k} \\
& \propto \exp \left\{-\frac{\left|r_{k}-m_{\omega_{k}}^{\downarrow}-m_{c_{k}}^{\downarrow} e^{j \theta_{k}}\right|^{2}}{2\left(v_{\omega_{k}}^{\downarrow}+v_{c_{k}}^{\downarrow}\right)}\right\}
\end{aligned}
$$

Using the following property of Tikhonov distribution, i.e.,

$$
g_{\mathcal{C}}\left(x e^{j \theta}, \sigma^{2} ; r\right) \propto I_{0}\left(\left|\frac{2 r x^{*}}{\sigma^{2}}\right|\right) t\left(\frac{2 r x^{*}}{\sigma^{2}} ; \theta\right)
$$

where $t\left(\frac{2 r x^{*}}{\sigma^{2}} ; \theta\right)$ represents Tikhonov distribution of variable $\theta$, we have

$$
p_{d}\left(\theta_{k}\right) \propto t\left(\gamma_{k} ; \theta_{k}\right)
$$

with parameter $\gamma_{k}$ as

$$
\gamma_{k} \triangleq \frac{2\left(r_{k}-m_{\omega_{k}}^{\downarrow}\right) m_{c_{k}}^{\downarrow *}}{v_{\omega_{k}}^{\downarrow}+v_{c_{k}}^{\downarrow}} .
$$

Denote the forward and backward messages from $p\left(\theta_{k} \mid \theta_{k-1}, \varphi\right)$ and $p\left(\theta_{k+1} \mid \theta_{k}, \varphi\right)$ to variable node $\theta_{k}$ as $p_{f}\left(\theta_{k}\right)$ and $p_{b}\left(\theta_{k}\right)$. In order to achieve low-complexity parametric message passing, we further constrain the two messages to be Tikhonov distributions ${ }^{3}$, i.e., $p_{f}\left(\theta_{k}\right) \propto t\left(a_{f, k} ; \theta_{k}\right), p_{b}\left(\theta_{k}\right) \propto t\left(a_{b, k} ; \theta_{k}\right)$. Then,

\footnotetext{
${ }^{3}$ It will be shown later that based on the expression of $p_{d}\left(\theta_{k}\right)$ in (12), the constraint of Tikhonov distributions of $p_{f}\left(\theta_{k}\right)$ and $p_{b}\left(\theta_{k}\right)$ are reasonable.
} 
we can calculate the message from $p\left(\theta_{k} \mid \theta_{k-1}, \varphi\right)$ to the variable node $\varphi$ as

$$
\begin{aligned}
\mu_{p\left(\theta_{k} \mid \theta_{k-1}, \varphi\right) \rightarrow \varphi} & \propto \iint p_{d}\left(\theta_{k}\right) p_{b}\left(\theta_{k}\right) p_{d}\left(\theta_{k-1}\right) p_{f}\left(\theta_{k-1}\right) g\left(\theta_{k-1}+\varphi, \sigma_{\varepsilon}^{2} ; \theta_{k}\right) \mathrm{d} \theta_{k-1} \mathrm{~d} \theta_{k} \\
& \propto \iint t\left(\gamma_{k}+a_{b, k} ; \theta_{k}\right) t\left(\gamma_{k-1}+a_{f, k-1} ; \theta_{k-1}\right) g\left(\theta_{k-1}+\varphi, \sigma_{\varepsilon}^{2} ; \theta_{k}\right) \mathrm{d} \theta_{k-1} \mathrm{~d} \theta_{k} \\
& \propto \frac{I_{0}\left(\left|\gamma_{k}+a_{b, k}+\frac{\left(\gamma_{k-1}+a_{f, k-1}\right) e^{j \varphi}}{1+\sigma_{\varepsilon}^{2}\left|\left(\gamma_{k-1}+a_{f, k-1}\right) e^{j \varphi}\right|}\right|\right)}{I_{0}\left(\left|\frac{\left(\gamma_{k-1}+a_{f, k-1}\right) e^{j \varphi}}{1+\sigma_{\varepsilon}^{2}\left|\left(\gamma_{k-1}+a_{f, k-1}\right) e^{j \varphi}\right|}\right|\right)}
\end{aligned}
$$

Then we can calculate the message from variable $\varphi$ to $p\left(\theta_{k} \mid \theta_{k-1}, \varphi\right)$, denoted as $\mu_{\varphi \rightarrow p\left(\theta_{k} \mid \theta_{k-1}, \varphi\right)}$, based on the SPA rules. However, since the messages $\mu_{\varphi \rightarrow p\left(\theta_{k} \mid \theta_{k-1}, \varphi\right)}$ for $k \in\{1, \cdots, K\}$ are different and have to be calculated separately, the complexity is huge. We propose to use Dirac delta function $\delta(\varphi-\hat{\varphi})$ to approximate these messages, where $\hat{\varphi}$ is the MAP estimation of $\varphi$, which is given by

$$
\hat{\varphi}=\underset{\varphi}{\operatorname{argmax}}(\underbrace{p(\varphi) \prod_{k} \mu_{p\left(\theta_{k} \mid \theta_{k-1}, \varphi\right) \rightarrow \varphi}}_{b(\varphi)}),
$$

where $p(\varphi)$ is the prior distribution of $\varphi$. Without loss of generality, we assume $p(\varphi)$ is a uniform distribution. Substituting (16) into (17), there is no closed-form solution and we have to resort to numerical method.

The conjugate gradient $(\mathrm{CG})$ method is useful to solve unconstrained optimization problems, and the convergence speed of CG is faster than that of the gradient descent (GD) method. The update rule of $\mathrm{CG}$ is:

$$
\hat{\varphi}_{n+1}=\hat{\varphi}_{n}-\iota d_{n}
$$

where $n$ is the index of inner iteration, $\iota>0$ denotes the step size, $d_{n}$ is the 
search direction given by

$$
d_{n}=\left\{\begin{array}{ll}
-g_{n} & n=0 \\
-g_{n}+\beta_{n} d_{n-1} & n \geqslant 1
\end{array} .\right.
$$

where $\beta_{n}=\frac{g_{n}^{T}\left(g_{n}-g_{n-1}\right)}{\left\|g_{n-1}\right\|^{2}}$ is the conjugate gradient coefficient [25], $g_{n}$ is the gradient of $\ln b(\varphi)$, i.e.,

$$
\begin{aligned}
g_{n}= & \sum_{k=1}^{K}\left\{\frac{j\left(\gamma_{k-1}+a_{f, k-1}\right) e^{j \varphi_{n}}}{\left(1+\sigma_{\varepsilon}^{2}\left|\left(\gamma_{k-1}+a_{f, k-1}\right) e^{j \varphi_{n}}\right|\right)^{2}}\right. \\
& \left.\times\left[\frac{I_{1}\left(\left|\frac{\left(\gamma_{k-1}+a_{f, k-1}\right) e^{j \varphi_{n}}}{1+\sigma_{\varepsilon}^{2}\left|\left(\gamma_{k-1}+a_{f, k-1}\right) e^{j \varphi_{n}}\right|}\right|\right)}{I_{0}\left(\left|\frac{\left(\gamma_{k-1}+a_{f, k-1}\right) e^{j \varphi_{n}}}{1+\sigma_{\varepsilon}^{2}\left|\left(\gamma_{k-1}+a_{f, k-1}\right) e^{j \varphi_{n}}\right|}\right|\right)}-\frac{I_{1}\left(\left|\gamma_{k}+a_{b, k}+\frac{\left(\gamma_{k-1}+a_{f, k-1}\right) e^{j \varphi_{n}}}{1+\sigma_{\varepsilon}^{2}\left|\left(\gamma_{k-1}+a_{f, k-1}\right) e^{j \varphi_{n} \mid}\right|}\right|\right)}{I_{0}\left(\left|\gamma_{k}+a_{b, k}+\frac{\left(\gamma_{k-1}+a_{f, k-1}\right) e^{j \varphi_{n}}}{1+\sigma_{\varepsilon}^{2}\left|\left(\gamma_{k-1}+a_{f, k-1}\right) e^{j \varphi_{n}}\right|}\right|\right)}\right]\right\} .
\end{aligned}
$$

After having the MAP estimation $\hat{\varphi}$, we can calculate the forward message $p_{f}\left(\theta_{k+1}\right)$ as

$$
\begin{aligned}
p_{f}\left(\theta_{k+1}\right) & =\iint_{f} p_{f}\left(\theta_{k}\right) p_{d}\left(\theta_{k}\right) p\left(\theta_{k+1} \mid \theta_{k}, \varphi\right) \delta(\varphi-\hat{\varphi}) \mathrm{d} \theta_{k} \mathrm{~d} \varphi \\
& \propto \int_{-\pi}^{\pi} p_{f}\left(\theta_{k}\right) t\left(\gamma_{k} ; \theta_{k}\right) g\left(\theta_{k}+\hat{\varphi}, \sigma_{\varepsilon}^{2} ; \theta_{k+1}\right) \mathrm{d} \theta_{k} .
\end{aligned}
$$

It can be observed from the recursive function in $(21)$ that $p_{f}\left(\theta_{k+1}\right)$ are in the form of Tikhonov distribution, i.e., $p_{f}\left(\theta_{k+1}\right) \propto t\left(a_{f, k+1} ; \theta_{k+1}\right)$. Substituting the Tikhonov expression into (21), the parameter can be recursively calculated as

$$
a_{f, k+1}=\frac{\gamma_{k}+a_{f, k}}{1+\sigma_{\varepsilon}^{2}\left|\gamma_{k}+a_{f, k}\right|} e^{j \hat{\varphi}}
$$

In a similar way, we have the backward message $p_{b}\left(\theta_{k-1}\right) \propto t\left(a_{b, k-1} ; \theta_{k-1}\right)$ with parameter recursively updated as

$$
a_{b, k-1}=\frac{a_{b, k}+\gamma_{k}}{1+\sigma_{\varepsilon}^{2}\left|a_{b, k}+\gamma_{k}\right|} e^{-j \hat{\varphi}} .
$$


Finally, the outgoing message of PHN and CFO subgraph $p_{u}\left(\theta_{k}\right)$ is given by

$$
\begin{aligned}
p_{u}\left(\theta_{k}\right) & =p_{f}\left(\theta_{k}\right) p_{b}\left(\theta_{k}\right) \\
& \propto \frac{I_{0}\left(\left|a_{f, k}+a_{b, k}\right|\right)}{2 \pi I_{0}\left(\left|a_{f, k}\right|\right) I_{0}\left(\left|a_{b, k}\right|\right)} t\left(a_{f, k}+a_{b, k} ; \theta_{k}\right) .
\end{aligned}
$$

\subsection{Messages on the Subgraph of FTN Symbol Detection}

Having the message $p_{u}\left(\theta_{k}\right)$ from PHN and CFO subgraph, we are able to calculate the expressions of $p_{u}\left(c_{k}\right)$ and $p_{u}\left(\omega_{k}\right)$, and then update all the messages on the subgraph of FTN symbol detection.

Based on SPA rules, the message $p_{u}\left(c_{k}\right)$ can be calculated as

$$
\begin{aligned}
p_{u}\left(c_{k}\right) & =\iint p_{u}\left(\theta_{k}\right) p_{d}\left(\omega_{k}\right) p\left(r_{k} \mid c_{k}, \theta_{k}, \omega_{k}\right) \mathrm{d} \omega_{k} \mathrm{~d} \theta_{k} \\
& \propto \exp \left\{-\frac{\left|c_{k}\right|^{2}}{2 v_{\omega_{k}}^{\downarrow}}\right\} \int t\left(a_{f, k} ; \theta_{k}\right) t\left(a_{b, k} ; \theta_{k}\right) \exp \left\{\Re \left[\frac{\left(r_{k}-m_{\omega_{k}}^{\downarrow}\right) c_{k}^{*}}{v_{\omega_{k}}^{\downarrow}} e^{\left.-j \theta_{k}\right]} \mathrm{d} \theta_{k}\right.\right. \\
& \propto \exp \left\{-\frac{\left|c_{k}\right|^{2}}{2 v_{\omega_{k}}^{\downarrow}}\right\} \frac{I_{0}\left(\left|a_{f, k}+a_{b, k}+\frac{\left(r_{k}-m_{\omega_{k}}^{\downarrow}\right) c_{k}^{*}}{v_{\omega_{k}}^{\downarrow}}\right|\right)}{2 \pi I_{0}\left(\left|a_{f, k}\right|\right) I_{0}\left(\left|a_{b, k}\right|\right)}
\end{aligned}
$$

However, it is not efficient to use the expression of $p_{u}\left(c_{k}\right)$ in (25) to update the other messages directly, since no closed-form representation of messages can be obtained and one has to resort to sampling method [18], which involves high complexity. We propose two methods to solve this problem. The first one aims at approximating $p_{u}\left(c_{k}\right)$ in Gaussian form via minimizing KLD between the distributions. In the second method, we develop a combined SPVMP algorithm. Both of the methods lead to efficient parametric message passing. 


\subsubsection{Gaussian Approximation via KLD Minimization}

KLD is a measure of the difference between two probability distributions $P$ and $Q$ denoted by $D_{K L}(P \| Q)[26]$. Generally, $P$ represents the actual distribution, while $Q$ is an approximation of $P$. Assuming $p(x)$ and $q(x)$ denote the densities of $P$ and $Q$, respectively, the KLD is defined as [27]:

$$
D_{K L}(P \| Q)=\int_{-\infty}^{+\infty} p(x) \log \frac{p(x)}{q(x)} \mathrm{d} x .
$$

We can approximate $p_{u}\left(c_{k}\right)$ by $\tilde{p}_{u}\left(c_{k}\right) \triangleq g_{\mathcal{C}}\left(m_{c_{k}}^{\uparrow}, v_{c_{k}}^{\uparrow} ; c_{k}\right)$ via minimizing the KLD. The optimal parameters $m_{c_{k}}^{\uparrow}$ and $v_{c_{k}}^{\uparrow}$ are given by

$$
\left(m_{c_{k}}^{\uparrow}, v_{c_{k}}^{\uparrow}\right)=\underset{\left(m_{c_{k},}, v_{c_{k}}\right)}{\operatorname{argmin}} D_{K L}\left(p_{u}\left(c_{k}\right) \mid \tilde{p}_{u}\left(c_{k}\right)\right) .
$$

We remark that (27) is equivalent to the moment matching between $p_{u}\left(c_{k}\right)$ and $\tilde{p}_{u}\left(c_{k}\right)$, which can be solved by numerical method. Then, based on the Gaussian approximation of $p_{u}\left(c_{k}\right)$, we are able to represent messages on the FTN symbol detection subgraph in Gaussian forms. Accordingly, only the mean and variance of the message have to be updated, which significantly reduces the computational complexity.

\subsubsection{Combined SP-VMP Method}

Since numerical method has to be employed in KLD minimization, the complexity is still high. We develop a combined SP-VMP algorithm to tackle this problem.

First, we modify the factor graph around the factor node $p\left(r_{k} \mid c_{k}, \theta_{k}, \omega_{k}\right)$, which is illustrated in Fig. 3. A virtual prior of colored noise $\omega_{k}$ is included, denoted as $p_{d}\left(\omega_{k}\right)$. Then, the nodes in the dashed box on the left hand side 
of Fig. 3 are grouped to construct a new factor node $p\left(r_{k} \mid c_{k}, \theta_{k}\right)$, which can be expressed as

$$
\begin{aligned}
p\left(r_{k} \mid c_{k}, \theta_{k}\right) & \propto \exp \left\{-\frac{\left|r_{k}-m_{\omega_{k}}-c_{k} e^{j \theta_{k}}\right|^{2}}{2 v_{\omega_{k}}}\right\} \\
& \propto \exp \left\{-\frac{\left|c_{k}\right|^{2}}{2 v_{\omega_{k}}}\right\} \exp \left\{\Re\left[\frac{\left(r_{k}-m_{\omega_{k}}\right) c_{k}^{*}}{v_{\omega_{k}}} e^{-j \theta_{k}}\right]\right\},
\end{aligned}
$$

where the parameters $m_{\omega_{k}}$ and $v_{\omega_{k}}$ are determined by calculating the message $p_{u}\left(\omega_{k}\right) p_{d}\left(\omega_{k}\right) \propto g_{\mathcal{C}}\left(m_{\omega_{k}}, v_{\omega_{k}} ; \omega_{k}\right)$, which gives

$$
\begin{aligned}
v_{\omega_{k}} & =\left(v_{\omega_{k}}^{\uparrow-1}+v_{\omega_{k}}^{\downarrow-1}\right)^{-1} \\
m_{\omega_{k}} & =v_{\omega_{k}}\left(v_{\omega_{k}}^{\uparrow-1} m_{\omega_{k}}^{\uparrow}+v_{\omega_{k}}^{\downarrow-1} m_{\omega_{k}}^{\downarrow}\right)
\end{aligned}
$$

Instead of using SPA, we employ VMP to calculate the message $p_{u}\left(c_{k}\right)$. Based on the VMP rules [28], $p_{u}\left(c_{k}\right)$ is given by

$$
p_{u}\left(c_{k}\right)=\exp \left\{\int p_{u}\left(\theta_{k}\right) p_{d}\left(\theta_{k}\right) \ln p\left(r_{k} \mid c_{k}, \theta_{k}\right) \mathrm{d} \theta_{k}\right\} .
$$

Substituting (14), (24) and (28) into (31), after some manipulations (see Appendix A for details), we have

$$
p_{u}\left(c_{k}\right) \propto g_{\mathcal{C}}\left(m_{c_{k}}^{\uparrow}, v_{c_{k}}^{\uparrow} ; c_{k}\right)
$$

with

$$
\begin{aligned}
& m_{c_{k}}^{\uparrow}=\frac{I_{1}\left(\left|a_{f, k}+a_{b, k}+\gamma_{k}\right|\right)\left(r_{k}-m_{\omega_{k}}\right)}{2 I_{0}\left(\left|a_{f, k}+a_{b, k}+\gamma_{k}\right|\right)}, \\
& v_{c_{k}}^{\uparrow}=\frac{4 \pi^{2} I_{0}\left(\left|a_{f, k}\right|\right) I_{0}\left(\left|a_{b, k}\right|\right) I_{0}\left(\left|\gamma_{k}\right|\right) v_{\omega_{k}}}{I_{0}\left(\left|a_{f, k}+a_{b, k}+\gamma_{k}\right|\right)} .
\end{aligned}
$$

It is observed that, using VMP rules on the constructed factor node $p\left(r_{k} \mid c_{k}, \theta_{k}\right)$, the message $p_{u}\left(c_{k}\right)$ can be represented by Gaussian distribution. 
Considering the linear model in the subgraph of FTN symbol detection, we can perform SPA on the other nodes to achieve Gaussian parametric message passing. Compared to the KLD minimization method, the proposed combined SP-VMP algorithm provides closed-form solution, which results in lower computational complexity.

Similar to the method used to calculate $p_{u}\left(c_{k}\right)$, we can modify the factor graph and apply VMP to obtain $p_{u}\left(\omega_{k}\right)$ as

$$
\begin{aligned}
p_{u}\left(\omega_{k}\right) & =\exp \left\{\int p_{u}\left(\theta_{k}\right) p_{d}\left(\theta_{k}\right) \ln p\left(r_{k} \mid \theta_{k}, \omega_{k}\right) \mathrm{d} \theta_{k}\right\} \\
& \propto g_{\mathcal{C}}\left(m_{\omega_{k}}^{\uparrow}, v_{\omega_{k}}^{\uparrow} ; \omega_{k}\right),
\end{aligned}
$$

with

$$
\begin{aligned}
m_{\omega_{k}}^{\uparrow} & =r_{k}-\frac{I_{1}\left(\left|a_{f, k}+a_{b, k}+\gamma_{k}\right|\right) m_{c_{k}}}{2 I_{0}\left(\left|a_{f, k}+a_{b, k}+\gamma_{k}\right|\right)} \\
v_{\omega_{k}}^{\uparrow} & =\frac{4 \pi^{2} I_{0}\left(\left|a_{f, k}\right|\right) I_{0}\left(\left|a_{b, k}\right|\right) I_{0}\left(\left|\gamma_{k}\right|\right) v_{c_{k}}}{I_{0}\left(\left|a_{f, k}+a_{b, k}+\gamma_{k}\right|\right)} .
\end{aligned}
$$

where the parameters $m_{c_{k}}$ and $v_{c_{k}}$ are determined by calculating the message $p_{u}\left(c_{k}\right) p_{d}\left(c_{k}\right) \propto g_{\mathcal{C}}\left(m_{c_{k}}, v_{c_{k}} ; c_{k}\right)$, which gives

$$
\begin{aligned}
v_{c_{k}} & =\left(v_{c_{k}}^{\uparrow-1}+v_{c_{k}}^{\downarrow-1}\right)^{-1} \\
m_{c_{k}} & =v_{c_{k}}\left(v_{c_{k}}^{\uparrow-1} m_{c_{k}}^{\uparrow}+v_{c_{k}}^{\downarrow-1} m_{c_{k}}^{\downarrow}\right)
\end{aligned}
$$

Based on the Gaussian representation of $p_{u}\left(\omega_{k}\right)$, using the SPA rules, we can derive the forward and backward message $p_{f}\left(\omega_{k+1}\right)$ and $p_{b}\left(\omega_{k}\right)$ in Gaussian forms, i.e., $p_{f}\left(\omega_{k+1}\right) \propto g_{\mathcal{C}}\left(m_{f \omega_{k+1}}, v_{f \omega_{k+1}} ; \omega_{k+1}\right)$ and $p_{b}\left(\omega_{k}\right) \propto g_{\mathcal{C}}\left(m_{b \omega_{k}}, v_{b \omega_{k}} ; \omega_{k}\right)$, with parameters updated as follows

$$
m_{f \omega_{k+1}}=\alpha\left(\frac{1}{v_{f \omega_{k}}}+\frac{1}{v_{\omega_{k}}^{\uparrow}}\right)^{-1}\left(\frac{m_{f \omega_{k}}}{v_{f \omega_{k}}}+\frac{m_{\omega_{k}}^{\uparrow}}{v_{\omega_{k}}^{\uparrow}}\right)
$$




$$
\begin{gathered}
v_{f \omega_{k+1}}=\sigma_{\epsilon}^{2}+\alpha^{2}\left(\frac{1}{v_{f \omega_{k}}}+\frac{1}{v_{\omega_{k}}^{\uparrow}}\right)^{-1} \\
m_{b \omega_{k}}=\left(\frac{1}{v_{b \omega_{k+1}}}+\frac{1}{v_{\omega_{k+1}}^{\uparrow}}\right)^{-1}\left(\frac{m_{b \omega_{k+1}}}{v_{b \omega_{k+1}}}+\frac{m_{\omega_{k+1}}^{\uparrow}}{v_{\omega_{k+1}}^{\uparrow}}\right) / \alpha, \\
v_{b \omega_{k}}=\left[\sigma_{\epsilon}^{2}+\left(\frac{1}{v_{b \omega_{k+1}}}+\frac{1}{v_{\omega_{k+1}}^{\uparrow}}\right)^{-1}\right] / \alpha^{2} .
\end{gathered}
$$

Accordingly, we have

$$
p_{d}\left(\omega_{k}\right)=p_{f}\left(\omega_{k}\right) p_{b}\left(\omega_{k}\right) \propto g_{\mathcal{C}}\left(m_{\omega_{k}}^{\downarrow}, v_{\omega_{k}}^{\downarrow} ; \omega_{k+1}\right)
$$

with parameters as

$$
\begin{gathered}
m_{\omega_{k}}^{\downarrow}=\frac{m_{\omega_{k}}^{\uparrow} v_{f \omega_{k}} v_{b \omega_{k}}+\left(m_{f \omega_{k}} v_{b \omega_{k}}+m_{b \omega_{k}} v_{f \omega_{k}}\right) v_{\omega_{k}}^{\uparrow}}{v_{f \omega_{k}} v_{b \omega_{k}}+\left(v_{f \omega_{k}}+v_{b \omega_{k}}\right) v_{\omega_{k}}^{\uparrow}} \\
v_{\omega_{k}}^{\downarrow}=\frac{v_{f \omega_{k}} v_{b \omega_{k}} v_{\omega_{k}}^{\uparrow}}{v_{f \omega_{k}} v_{b \omega_{k}}+\left(v_{f \omega_{k}}+v_{b \omega_{k}}\right) v_{\omega_{k}}^{\uparrow}}
\end{gathered}
$$

Having the expression of $p_{u}\left(c_{k}\right)$, we can now further derive the message $p_{u}\left(x_{k}\right)$ passed to the channel decoder. First, we calculate the message from the variable nodes $\mathbf{s}_{k-1}$ and $\mathbf{s}_{k}$ to the function node $f_{\mathbf{s}_{k}}$, denoted by $p_{f}\left(\mathbf{s}_{k-1}\right)$ and $p_{b}\left(\mathbf{s}_{k}\right)$, respectively. Assuming $p_{d}\left(x_{k+1}\right)=g_{\mathcal{C}}\left(m_{x_{k+1}}^{\downarrow}, v_{x_{k+1}}^{\downarrow} ; x_{k+1}\right)$ and $p_{b}\left(\mathbf{s}_{k+1}\right)=g_{\mathcal{C}}\left(\mathbf{m}_{b \mathbf{s}_{k+1}}, \mathbf{V}_{b \mathbf{s}_{k+1}} ; \mathbf{s}_{k+1}\right)$, we can calculate the message $\mu_{f_{\mathbf{s}_{k+1} \rightarrow \mathbf{s}_{k}}}\left(\mathbf{s}_{k}\right)$ by SPA as

$$
\begin{aligned}
\mu_{\mathbf{s}_{k+1} \rightarrow \mathbf{s}_{k}}\left(\mathbf{s}_{k}\right) & =\iint f_{\mathbf{s}_{k+1}} p_{d}\left(x_{k+1}\right) p_{b}\left(\mathbf{s}_{k+1}\right) \mathrm{d} x_{k+1} \mathrm{~d} \mathbf{s}_{k+1} \\
& =\iint f_{\mathbf{s}_{k+1}} g_{\mathcal{C}}\left(m_{x_{k+1}}^{\downarrow}, v_{x_{k+1}}^{\downarrow} ; x_{k+1}\right) g_{\mathcal{C}}\left(\mathbf{m}_{b \mathbf{s}_{k+1}}, \mathbf{V}_{b \mathbf{s}_{k+1}} ; \mathbf{s}_{k+1}\right) \mathrm{d} x_{k+1} \mathrm{~d} \mathbf{s}_{k+1}
\end{aligned}
$$


Since $f_{\mathbf{s}_{k+1}}=\delta\left(\mathbf{s}_{k+1}-\boldsymbol{\Lambda} \mathbf{s}_{k}-\mathbf{a} x_{k+1}\right)$, we can obtain $\mu_{f_{\mathbf{s}_{k+1}} \rightarrow \mathbf{s}_{k}}\left(\mathbf{s}_{k}\right) \propto g_{\mathcal{C}}\left(\mathbf{m}_{b \mathbf{s}_{k}}^{\prime \prime}, \mathbf{W}_{b \mathbf{s}_{k}}^{\prime \prime} ; \mathbf{s}_{k}\right)$, where

$$
\begin{gathered}
\mathbf{W}_{b \mathbf{s}_{k}}^{\prime \prime} m_{b \mathbf{s}_{k}}^{\prime \prime}=\Lambda^{H}\left(\mathbf{V}_{b \mathbf{s}_{k+1}}+\mathbf{a} v_{x_{k+1}}^{\downarrow} \mathbf{a}^{H}\right)^{-1}\left(\mathbf{m}_{b \mathbf{s}_{k+1}}-\mathbf{a} m_{x_{k+1}}^{\downarrow}\right), \\
\mathbf{W}_{b \mathbf{s}_{k}}^{\prime \prime}=\Lambda^{H}\left(\mathbf{V}_{b \mathbf{s}_{k+1}}+\mathbf{a} v_{x_{k+1}}^{\downarrow} \mathbf{a}^{H}\right)^{-1} \boldsymbol{\Lambda} .
\end{gathered}
$$

Then, the message $p_{b}\left(\mathbf{s}_{k}\right)$ is given by

$$
\begin{aligned}
p_{b}\left(\mathbf{s}_{k}\right) & =\mu_{f_{\mathbf{s}_{k+1}} \rightarrow \mathbf{s}_{k}} \int \delta\left(c_{k}-\mathbf{h}^{T} \mathbf{s}_{k}\right) p_{u}\left(c_{k}\right) \mathrm{d} c_{k} \\
& \propto \exp \left\{-\left(\mathbf{s}_{k}-\mathbf{m}_{b \mathbf{s}_{k}}\right)^{H} \mathbf{V}_{b \mathbf{s}_{k}}^{-1}\left(\mathbf{s}_{k}-\mathbf{m}_{b \mathbf{s}_{k}}\right)\right\},
\end{aligned}
$$

with

$$
\begin{gathered}
\mathbf{V}_{b \mathbf{s}_{k}}=\left[\mathbf{W}_{b \mathbf{s}_{k}}^{\prime \prime}+\mathbf{h} v_{c_{k}}^{\uparrow-1} \mathbf{h}^{H}\right]^{-1} \\
\mathbf{m}_{b \mathbf{s}_{k}}=\mathbf{V}_{b \mathbf{s}_{k}}\left[\mathbf{W}_{b \mathbf{s}_{k}}^{\prime \prime} \mathbf{m}_{b \mathbf{s}_{k}}^{\prime \prime}+\mathbf{h} v_{c_{k}}^{\uparrow-1} m_{c_{k}}^{\uparrow}\right] .
\end{gathered}
$$

Similar to that in (47) and (50), we can calculate the expressions of $\mu_{f_{\mathbf{s}_{k-1} \rightarrow} \mathbf{s}_{k-1}}\left(\mathbf{s}_{k-1}\right)=g_{\mathcal{C}}\left(\mathbf{m}_{f \mathbf{s}_{k-1}}^{\prime \prime}, \mathbf{V}_{f \mathbf{s}_{k-1}}^{\prime \prime} ; \mathbf{s}_{k-1}\right)$ and $p_{f}\left(\mathbf{s}_{k-1}\right)=g_{\mathcal{C}}\left(\mathbf{m}_{f \mathbf{s}_{k-1}}, \mathbf{V}_{f \mathbf{s}_{k-1}} ; \mathbf{s}_{k-1}\right)$, with parameters as follows

$$
\begin{gathered}
\mathbf{V}_{f \mathbf{s}_{k-1}}^{\prime \prime}=\Lambda \mathbf{V}_{f \mathbf{s}_{k-2}} \Lambda^{H}+\mathbf{a} v_{x_{k-1}}^{\downarrow} \mathbf{a}^{H} \\
\mathbf{m}_{f \mathbf{s}_{k-1}}^{\prime \prime}=\Lambda \mathbf{m}_{f_{\mathbf{s}_{k-2}}}+\mathbf{a} m_{x_{k-1}}^{\downarrow},
\end{gathered}
$$

and

$$
\mathbf{V}_{f \mathbf{s}_{k-1}}=\left[\mathbf{V}_{f \mathbf{s}_{k-1}}^{\prime \prime}+\mathbf{h} v_{c_{k-1}}^{\uparrow-1} \mathbf{h}^{H}\right]^{-1}
$$




$$
\mathbf{m}_{f \mathbf{s}_{k-1}}=\mathbf{V}_{f \mathbf{s}_{k-1}}\left[\mathbf{V}_{f \mathbf{s}_{k-1}}^{\prime \prime} \mathbf{m}_{f \mathbf{s}_{k-1}}^{\prime \prime}+\mathbf{h} v_{c_{k-1}-1}^{\uparrow} m_{c_{k-1}}^{\uparrow}\right]
$$

Finally, having the expressions of $p_{f}\left(\mathbf{s}_{k-1}\right)$ and $p_{b}\left(\mathbf{s}_{k}\right)$, the message $p_{u}\left(x_{k}\right)$ is given by

$$
\begin{aligned}
p_{u}\left(x_{k}\right) & =\int f_{\mathbf{s}_{k}} p_{f}\left(\mathbf{s}_{k-1}\right) p_{b}\left(\mathbf{s}_{k}\right) \mathrm{d} \mathbf{s}_{k} \mathrm{~d} \mathbf{s}_{k-1} \\
& \propto \exp \left\{-\frac{\left(x_{k}-m_{x_{k}}^{\uparrow}\right)^{2}}{2 v_{x_{k}}^{\uparrow}}\right\}
\end{aligned}
$$

where

$$
\begin{gathered}
m_{x_{k}}^{\uparrow}=\mathbf{a}^{H}\left(\mathbf{m}_{b \mathbf{s}_{k}}-\Lambda \mathbf{m}_{f \mathbf{s}_{k-1}}\right), \\
v_{x_{k}}^{\uparrow}=\mathbf{a}^{H}\left(\mathbf{V}_{b \mathbf{s}_{k}}+\Lambda \mathbf{V}_{f \mathbf{s}_{k-1}} \Lambda^{H}\right) \mathbf{a} .
\end{gathered}
$$

Then the updated message $p_{u}\left(x_{k}\right)$ is converted to log-likelihood ratio and fed to the channel decoder. The optimal decision $d_{k}$ is given by performing standard SPA decoding [29].

Then, the downward message $p_{d}\left(c_{k}\right)$ is given by

$$
\begin{aligned}
p_{d}\left(c_{k}\right) & =\int \mu_{f_{\mathbf{s}_{k}} \rightarrow \mathbf{s}_{k}}\left(\mathbf{s}_{k}\right) \mu_{f_{\mathbf{s}_{k+1}} \rightarrow \mathbf{s}_{k}}\left(\mathbf{s}_{k}\right) \delta\left(c_{k}-\mathbf{h}^{T} \mathbf{s}_{k}\right) \mathrm{d} \mathbf{s}_{k} \\
& =\int g_{\mathcal{C}}\left(\mathbf{m}_{\mathbf{s}_{k}}^{\downarrow}, \mathbf{V}_{\mathbf{s}_{k}}^{\downarrow} ; \mathbf{s}_{k}\right) \delta\left(c_{k}-\mathbf{h}^{T} \mathbf{s}_{k}\right) \mathrm{d} \mathbf{s}_{k} \\
& \propto g_{\mathcal{C}}\left(m_{c_{k}}^{\downarrow}, v_{c_{k}}^{\downarrow} ; c_{k}\right),
\end{aligned}
$$

where the parameters are given by

$$
\begin{aligned}
& \mathbf{V}_{\mathbf{s}_{k}}^{\downarrow}=\left[\mathbf{V}_{f \mathbf{s}_{k}}^{\prime \prime}+\mathbf{W}_{b \mathbf{s}_{k}}^{\prime \prime}\right]^{-1} \\
& \mathbf{m}_{\mathbf{s}_{k}}^{\downarrow}=\mathbf{V}_{\mathbf{s}_{k}}^{\downarrow}\left(\mathbf{V}_{f \mathbf{s}_{k}}^{\prime \prime}-1 \mathbf{m}_{f \mathbf{s}_{k}}^{\prime \prime}+\mathbf{W}_{b \mathbf{s}_{k}}^{\prime \prime} \mathbf{m}_{b \mathbf{s}_{k}}^{\prime \prime}\right),
\end{aligned}
$$


and

$$
\begin{aligned}
v_{c_{k}}^{\downarrow} & =\left[\left(\mathbf{h}^{T} \mathbf{V}_{\mathbf{s}_{k}}^{\downarrow} \mathbf{h}\right)^{-1}+v_{c_{k}}^{\uparrow-1}\right]^{-1}, \\
m_{c_{k}}^{\downarrow} & =v_{c_{k}}^{\downarrow}\left(\frac{\mathbf{h}^{T} \mathbf{m}_{\mathbf{s}_{k}}^{\downarrow}}{\mathbf{h}^{T} \mathbf{V}_{\mathbf{s}_{k}}^{\downarrow} \mathbf{h}}+\frac{m_{c_{k}}^{\uparrow}}{v_{c_{k}}^{\uparrow}}\right) .
\end{aligned}
$$

The proposed algorithm is summarized in Algorithm 1.

\section{Simulation Results and Discussion}

We evaluate the performance of the proposed algorithm and compare it with the state-of-the-art methods via Monte Carlo simulations. The serial scheduling method is employed due to it has the better performance than flooding scheduling [30]. Consider an LDPC-coded quadrature phase-shift keying (QPSK) modulation system with coding rate $r=5 / 7$ and code length $N=2016$. A root raised-cosine pulse shaping filter with roll-off factor $\alpha=$ 0.4 is employed. In each codeword, one pilot every 16 coded symbols has been inserted. The normalized CFO is $1.6 \times 10^{-2}$. The maximum number of iterations $^{4}$ is $I=20$.

The bit error rate (BER) performance of the proposed algorithm with different packing factor $\tau$ is illustrated in Fig. 4 and Fig. 5, where the standard deviations of the PHN $\sigma_{\Delta}=0.1^{\circ}$ and $\sigma_{\Delta}=3^{\circ}$, respectively. For comparison purpose, we also evaluate the performance of four scenarios: 1) Nyquist signaling in the presence of PHN and CFO (denoted by "Nyquist");

2) Joint PHN estimation and symbol detection in FTN signaling (denoted by "only equalization and PHN"). 3) Using the conventional maximum likelihood (ML) method to estimate the PHN, and LMMSE to handle the FTN

\footnotetext{
${ }^{4}$ We perform CG twice in each message passing iteration.
} 
signaling [11] (denoted by "ML and LMMSE"). 4) Symbol detection without considering PHN and CFO estimation (denoted by "only equalization"). We can observe from Fig. 4 and Fig. 5 that, ignoring PHN and CFO in Scenario 4 leads to significant performance loss, i.e., $1.5 \mathrm{~dB}$ for $\sigma_{\Delta}=0.1^{\circ}$ and $2 \mathrm{~dB}$ for $\sigma_{\Delta}=3^{\circ}$. The pilot-aided ML method in Scenario 3 can not provide accurate PHN estimation, which impacts the performance of the LMMSE detection of FTN signaling, especially when the PHN is strong. In Scenario 2, although PHN is estimated symbol by symbol, the signal model mismatch due to ignoring CFO still leads to about $1 \mathrm{~dB}$ performance loss. It is seen that the proposed algorithm for FTN detection can attain the Nyquist scenario with $\tau=0.8$ and 0.75 . When $\tau=0.6$, there exists $0.3 \mathrm{~dB}$ performance gap between the Nyquist and FTN signaling, while the latter can increase transmission rate up to $67 \%$. Moreover, the performance of the proposed two methods, namely, KLD and SP-VMP algorithm perform close to each other. Since the combined SP-VMP is able to update parameters of messages in closed-form expressions, the computational complexity is much lower.

We further evaluate the advantage of employing FTN signaling with different combinations of packing factor $\tau$ and coding rate $r$ in Fig. 6. Comparing the Nyquist signaling with $r=5 / 7$ and FTN signaling with $\tau=0.8$, $r=5 / 7$, we observe that they have almost the same BER performance, while the latter can provide about $25 \%$ higher spectral efficiency. On the other hand, if we fix the spectral efficiency to $\eta=1.02 \mathrm{bit} / \mathrm{s} / \mathrm{Hz}$, we can reduce both $\tau$ and $r$ to improve BER performance. It is seen that the gain is about $0.5 \mathrm{~dB}$ for $\tau=0.8, r=4 / 7,0.8 \mathrm{~dB}$ for $\tau=0.7, r=1 / 2$ and $1.1 \mathrm{~dB}$ for $\tau=0.57, r=2 / 5$ compared with the Nyquist signaling with $r=5 / 7$. 
However, if we keep decreasing $\tau$ and $r$, the BER performance may become worse, e.g., the gain reduces to $0.25 \mathrm{~dB}$ for $\tau=0.35, r=1 / 4$. This is due to the fact that, when $\tau$ is lower than the Mazo limit [1], the ISI imposed by FTN signaling can not be eliminated, and the coding gain by using lower rate channel code can not be fully exploited.

The mean squared errors (MSEs) of the PHN estimation with $\sigma_{\Delta}=0.1^{\circ}$ and $\sigma_{\Delta}=3^{\circ}$ are shown in Fig. 7 and Fig. 8. It is observed that pilot-aided ML estimation performs the worst since the data symbols are not employed. Moreover, we can see that the estimation accuracy of PHN degrades if CFO is not taking into account. The proposed two methods outperform the previous two methods by jointly considering the PHN and CFO estimation and FTN symbol detection. Especially, the proposed SP-VMP algorithm performs very close to the KLD method in estimation accuracy while providing closed-form expressions for parameters updating. We also evaluate the performance when there is no CFO or its impact is negligible. It is seen that the proposed SPVMP algorithm can also work in this scenario.

The BER performance of the proposed algorithm versus the number of iterations are shown in Fig. 9, with $\tau=0.8, \sigma=0.1^{\circ}$. As we can observe, the proposed algorithm converges after 10 iterations. However, after 5 iterations, the performance improvement by increasing the number of iterations becomes small. Therefore, we can trade-off between computational complexity and the BER performance in practice.

The MSEs of CFO estimation of the proposed algorithm using conjugate gradient (denoted by 'CG') and gradient decent (denoted by 'GD') are evaluated in Fig. 10. The method in [21] which is based on $Q$-level quantization 
of $\varphi$, is also plotted for comparison purpose. We can observe that the convergence speed of the method in [21] depends on the value of $Q$. However, since $Q$ parallel processing for quantized CFO values are required, large value of $Q$ leads to higher computational complexity. The proposed algorithm using CG converges faster than that with the traditional GD method, and both of them outperform the one in [21] with $Q=21$.

\section{Conclusions}

In this paper, we proposed iterative receiver for FTN signaling in the presence of PHN and CFO in a factor graph framework. Based on the efficient factorization of the joint posterior distribution, the factor graph was constructed, which consists two subgraphs, i.e., PHN and CFO estimation subgraph and the FTN symbol detection subgraph. Messages on the factor graph were derived using SPA rules, resulting in Tikhonov and Gaussian distributions on the two subgraphs, respectively. In order to perform parametric message passing, we proposed two methods to update message between subgraphs, namely, Gaussian approximation via KLD minimization and the combined SP-VMP method. It is seen that the proposed combined SP-VMP method is able to update parameters of Gaussian message in closed for$\mathrm{m}$, which reduced the computational complexity. Simulation results showed that the SP-VMP algorithm performed very close to the KLD minimization method, and both of them outperformed the existing methods in FTN signaling detection. Moreover, based on the proposed algorithm, FTN signaling (raised-cosine pulse with $\alpha=0.4$ ) can increase the transmission rate up to $67 \%$ compared with the Nyquist counterpart in the presence of PHN and 
CFO, with $E_{b} / N_{0}$ loss less than $0.3 \mathrm{~dB}$.

\section{Acknowledgment}

This work is supported by "National High Technology Research and Development Program of China" (Grant No. 2015AA01A709) and "National Science Foundation of China (NSFC)" (Grant 61201181 and Grant $61471037)$.

\section{Appendix}

Derivation of $p_{u}\left(c_{k}\right)$ based on VMP

Based on the VMP rules, the message $p_{u}\left(c_{k}\right)$ is given by

$$
\begin{aligned}
p_{u}\left(c_{k}\right)=\exp \{ & \left.\int p_{u}\left(\theta_{k}\right) p_{d}\left(\theta_{k}\right) \ln p\left(r_{k} \mid c_{k}, \theta_{k}\right) \mathrm{d} \theta_{k}\right\} \\
=\exp \{ & -\frac{I_{0}\left(\left|a_{f, k}+a_{b, k}+\gamma_{k}\right|\right)}{4 \pi^{2} I_{0}\left(\left|a_{f, k}\right|\right) I_{0}\left(\left|a_{b, k}\right|\right) I_{0}\left(\left|\gamma_{k}\right|\right)} \\
& \left.\times \int t\left(a_{f, k}+a_{b, k}+\gamma_{k} ; \theta_{k}\right) \frac{\mid r_{k}-m_{\omega_{k}}-c_{k} e^{\left.j \theta_{k}\right|^{2}}}{2 v_{\omega_{k}}} \mathrm{~d} \theta_{k}\right\} \\
=\exp \{ & -\frac{I_{0}\left(\left|a_{f, k}+a_{b, k}+\gamma_{k}\right|\right)}{4 \pi^{2} I_{0}\left(\left|a_{f, k}\right|\right) I_{0}\left(\left|a_{b, k}\right|\right) I_{0}\left(\left|\gamma_{k}\right|\right)}\left[\frac{\left|r_{k}-m_{\omega_{k}}\right|^{2}+\left|c_{k}\right|^{2}}{2 v_{\omega_{k}}}\right. \\
& \left.\left.-\frac{\Re\left\{\left(r_{k}-m_{\omega_{k}}\right)^{*} c_{k}\right\}}{v_{\omega_{k}}} \int t\left(a_{f, k}+a_{b, k}+\gamma_{k} ; \theta_{k}\right) \cos \left(\theta_{k}\right) d \theta_{k}\right]\right\}
\end{aligned}
$$

To calculate the integral, we can use the property of the Tikhonov distribution [31], i.e.,

$$
I_{n}(\kappa)=\frac{1}{\pi} \int_{0}^{\pi} e^{\kappa \cos \vartheta} \cos (n \vartheta) d \vartheta
$$

with $\vartheta \sim t(\zeta ; \vartheta), \zeta \triangleq \kappa e^{j \mu}$. The parameter $\kappa \geqslant 0$ represents the degree of

concentration, and $\mu \in[-\pi, \pi]$ decides the distribution mode. Then we can 
obtain

$$
\begin{aligned}
p_{u}\left(c_{k}\right)= & \exp \left\{-\frac{I_{0}\left(\left|a_{f, k}+a_{b, k}+\gamma_{k}\right|\right)}{4 \pi^{2} I_{0}\left(\left|a_{f, k}\right|\right) I_{0}\left(\left|a_{b, k}\right|\right) I_{0}\left(\left|\gamma_{k}\right|\right)}\right. \\
& \left.\times\left[\frac{\left|r_{k}-m_{\omega_{k}}\right|^{2}+\left|c_{k}\right|^{2}}{2 v_{\omega_{k}}}-\frac{\Re\left\{\left(r_{k}-m_{\omega_{k}}\right)^{*} c_{k}\right\}}{v_{\omega_{k}}} \frac{I_{1}\left(\left|a_{f, k}+a_{b, k}+\gamma_{k}\right|\right)}{2 I_{0}\left(\left|a_{f, k}+a_{b, k}+\gamma_{k}\right|\right)}\right]\right\}
\end{aligned}
$$

Denoting $A_{k}=\frac{I_{1}\left(\left|a_{f, k}+a_{b, k}+\gamma_{k}\right|\right)}{2 I_{0}\left(\left|a_{f, k}+a_{b, k}+\gamma_{k}\right|\right)}$ and $B_{k}=\frac{I_{0}\left(\left|a_{f, k}+a_{b, k}+\gamma_{k}\right|\right)}{4 \pi^{2} I_{0}\left(\left|a_{f, k}\right|\right) I_{0}\left(\left|a_{b, k}\right|\right) I_{0}\left(\left|\gamma_{k}\right|\right)}$, respectively, we have

$$
\begin{aligned}
p_{u}\left(c_{k}\right) & =\exp \left\{-B_{k}\left[\frac{\left|r_{k}-m_{\omega_{k}}\right|^{2}+\left|c_{k}\right|^{2}-2 A_{k} \Re\left[\left(r_{k}-m_{\omega_{k}}\right)^{*} c_{k}\right]}{2 v_{\omega_{k}}}\right]\right\} \\
& =\exp \left\{-\frac{\left|r_{k}-m_{\omega_{k}}\right|^{2}+\left|c_{k}\right|^{2}-2 A_{k} \Re\left[\left(r_{k}-m_{\omega_{k}}\right)^{*} c_{k}\right]}{2 \frac{v_{\omega_{k}}}{B_{k}}}\right\} \\
& \propto g_{\mathcal{C}}\left(m_{c_{k}}^{\uparrow}, v_{c_{k}}^{\uparrow} ; c_{k}\right)
\end{aligned}
$$

where

$$
\begin{gathered}
m_{c_{k}}^{\uparrow}=\frac{I_{1}\left(\left|a_{f, k}+a_{b, k}+\gamma_{k}\right|\right)\left(r_{k}-m_{\omega_{k}}\right)}{2 I_{0}\left(\left|a_{f, k}+a_{b, k}+\gamma_{k}\right|\right)} \\
v_{c_{k}}^{\uparrow}=\frac{4 \pi^{2} I_{0}\left(\left|a_{f, k}\right|\right) I_{0}\left(\left|a_{b, k}\right|\right) I_{0}\left(\left|\gamma_{k}\right|\right) v_{\omega_{k}}}{I_{0}\left(\left|a_{f, k}+a_{b, k}+\gamma_{k}\right|\right)}
\end{gathered}
$$

\section{Reference}

[1] J. Mazo, Faster-than-Nyquist signaling, Bell Syst. Tech. J. 54 (8) (1975) 1451-1462.

[2] J. B. Anderson, F. Rusek, V. Owall, Faster-than-Nyquist signaling, Proc. IEEE 101 (8) (2013) 1817-1830. 
[3] J. G. Andrews, S. Buzzi, W. Choi, S. V. Hanly, A. Lozano, A. C. Soong, J. C. Zhang, What will 5G be?, IEEE J. Sel. Areas Commun., 32 (6) (2014) 1065-1082.

[4] P. Banelli, S. Buzzi, G. Colavolpe, A. Modenini, F. Rusek, A. Ugolini, Modulation formats and waveforms for $5 \mathrm{G}$ networks: Who will be the heir of OFDM?: An overview of alternative modulation schemes for improved spectral efficiency, IEEE Signal Process. Mag. 31 (6) (2014) 80-93.

[5] J. E. Mazo, H. Landau, On the minimum distance problem for fasterthan-Nyquist signaling, IEEE Tran. Inform. Theory 34 (6) (1988) 1420 $-1427$.

[6] A. D. Liveris, C. N. Georghiades, Exploiting faster-than-Nyquist signaling, IEEE Tran. Commun. 51 (9) (2003) 1502-1511.

[7] F. Rusek, J. B. Anderson, Multistream faster than Nyquist signaling, IEEE Tran. Commun. 57 (5) (2009) 1329-1340.

[8] A. Prlja, J. B. Anderson, Reduced-complexity receivers for strongly narrowband intersymbol interference introduced by faster-than-Nyquist signaling, IEEE Tran. Commun. 60 (9) (2012) 2591-2601.

[9] S. Sugiura, Frequency-domain equalization of faster-than-Nyquist signaling, IEEE Wireless Commun. Lett. 2 (5) (2013) 555-558.

[10] S. Sugiura, L. Hanzo, Frequency-domain-equalization-aided iterative detection of faster-than-Nyquist signaling, IEEE Trans. Veh. Technol. 64 (5) (2015) $2122-2128$. 
[11] P. Şen, T. Aktaş, A. Ö. Yilmaz, A low-complexity graph-based LMMSE receiver designed for colored noise induced by FTN-signaling, in: Proc. Wireless Commun. Netw. Conf., IEEE, 2014, pp. 642-647.

[12] N. Noels, H. Steendam, M. Moeneclaey, Performance analysis of MLbased feedback carrier phase synchronizers for coded signals, IEEE Tran. Signal Process. 55 (3) (2007) 1129-1136.

[13] X. Wu, H. Xiang, Iterative carrier phase recovery methods in turbo receivers, IEEE Commun. Lett. 9 (8) (2005) 735 - 737.

[14] G. Colavolpe, A. Barbieri, G. Caire, Algorithms for iterative decoding in the presence of strong phase noise, IEEE J. Select.Areas Commun. 23 (9) (2005) $1748-1757$.

[15] M. Nissila, S. Pasupathy, Adaptive iterative detectors for phaseuncertain channels via variational bounding, IEEE Tran. Commun. 57 (3) (2009) $716-725$.

[16] D. D. Lin, T. J. Lim, The variational inference approach to joint data detection and phase noise estimation in OFDM, IEEE Tran. Signal Process. 55 (5) (2007) $1862-1874$.

[17] N. Noels, J. Bhatti, H. Bruneel, M. Moeneclaey, Block-processing softinput soft-output demodulator for coded PSK using DCT-based phase noise estimation, IEEE Tran. Commun. 62 (8) (2014) 2939 - 2950.

[18] F. R. Kschischang, B. J. Frey, H. A. Loeliger, Factor graphs and the sum-product algorithm, IEEE Tran. Inform. Theory 47 (2) (2001) 498519. 
[19] A. P. Worthen, W. E. Stark, Unified design of iterative receivers using factor graphs, IEEE Tran. Inform. Theory 47 (2) (2001) $843-849$.

[20] U.Mengali, Synchronization Techniques for Digital Receivers, Springer Science \& Business Media, 2013.

[21] A. Barbieri, G. Colavolpe, G. Caire, Joint iterative detection and decoding in the presence of phase noise and frequency offset, IEEE Tran. Commun. 55 (1) (2007) 171-179.

[22] A. Papoulis, Random Variables, and Stochastic Processes, McGraw-Hill, New York, 1990.

[23] U. N. Bhat, G. K. Miller, Elements of Applied Stochastic Processes, J. Wiley, 1972.

[24] N. Wu, W. Yuan, H. Wang, J. Kuang, Toa-based passive localization of multiple targets with inaccurate receivers based on belief propagation on factor graph, Digit. Signal Process. 49 (2016) 14-23.

[25] M. Mamat, M. Rivaie, I. Mohd, A new conjugate gradient coefficient for unconstrained optimization, Int. J. Contemp. Math. Sciences 5 (29) (2010) 1429-1437.

[26] S. Kullback, R. A. Leibler, On information and sufficiency, Ann. Math. Statist. 22 (1) (1951) 79-86.

[27] Y. Anzai, Pattern Recognition \& Machine Learning, Elsevier, 2012.

[28] J. Dauwels, On variational message passing on factor graphs, IEEE, 2007, pp. 2546-2550. 
[29] I. M. Tienda-Luna, D. P. Ruiz, Y. Huang, M. C. Carrión, Iterative decoding in factor graph representation using particle filtering, Digit. Signal Process. 22 (2) (2012) 212-218.

[30] G. Colavolpe, G. Germi, On the application of factor graphs and the sum-product algorithm to ISI channels, IEEE Tran. Commun. 53 (5) (2005) 818-825.

[31] M. Abramowitz, I. A. Stegun, et al., Handbook of Mathematical Functions, Applied Mathematics Series 55 (1966) 62. 


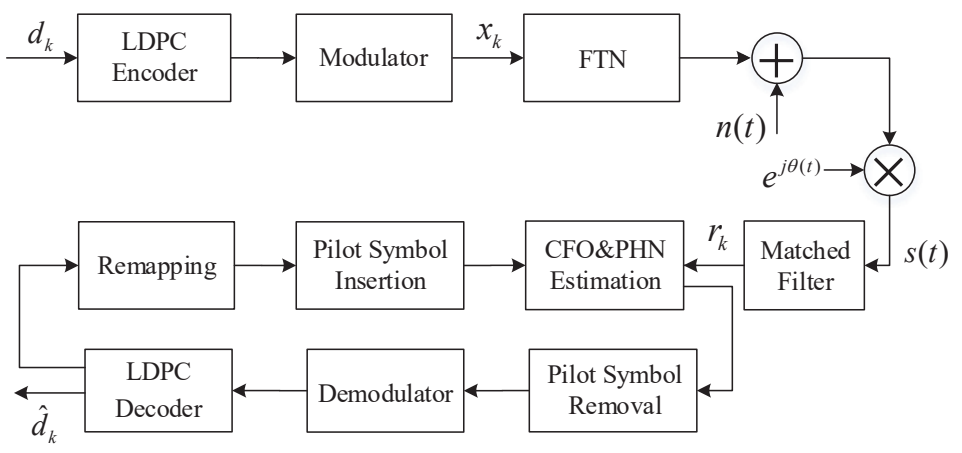

Figure 1: The block diagram of FTN system. 


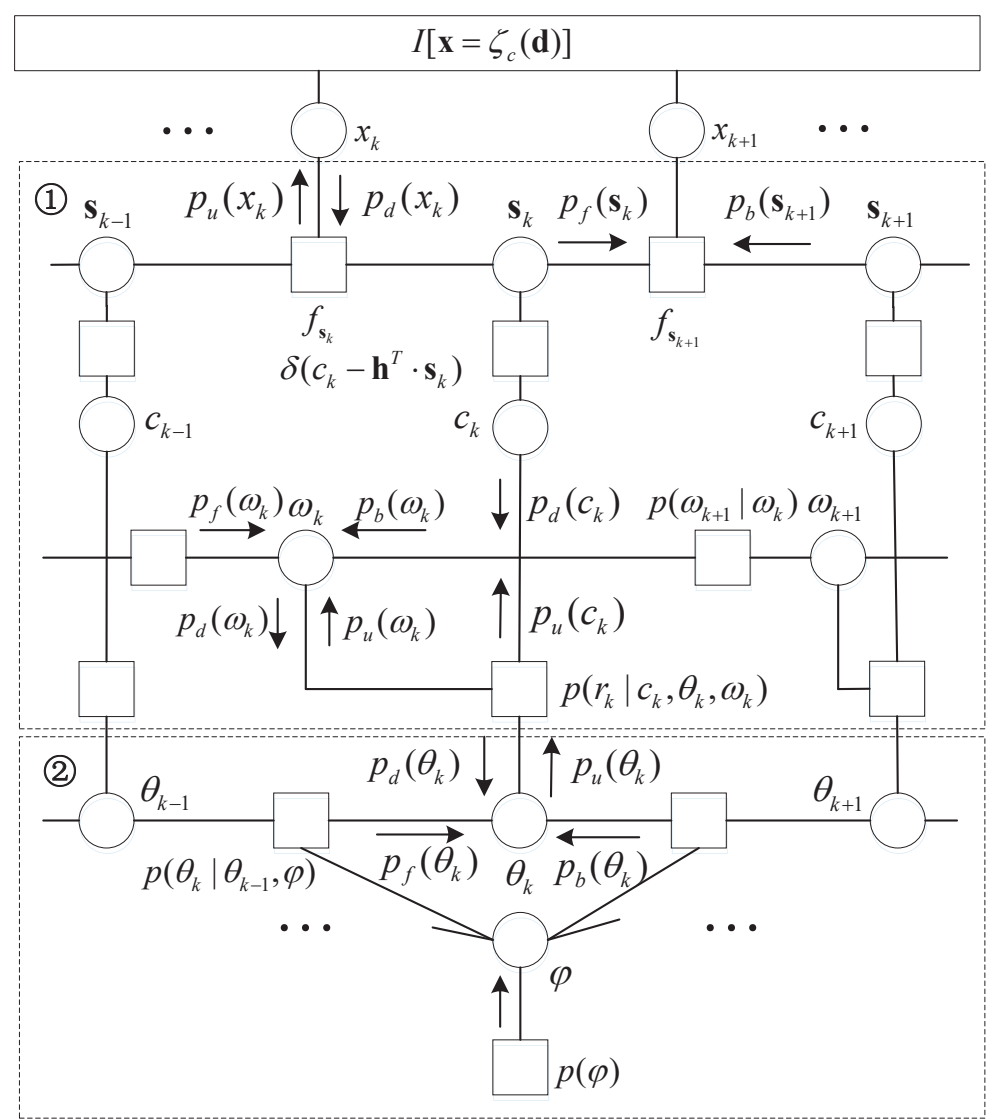

Figure 2: Factor graph model of FTN signaling detection in the presence of PHN and CFO: (1) FTN symbol detection subgraph (2) PHN and CFO estimation subgraph. 


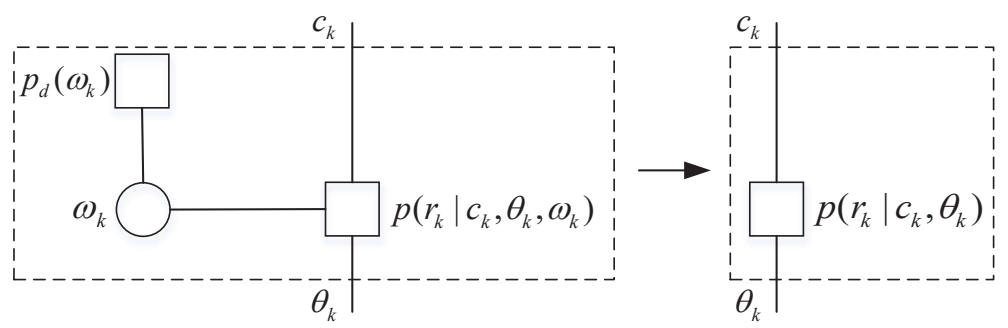

Figure 3: The modified factor graph.

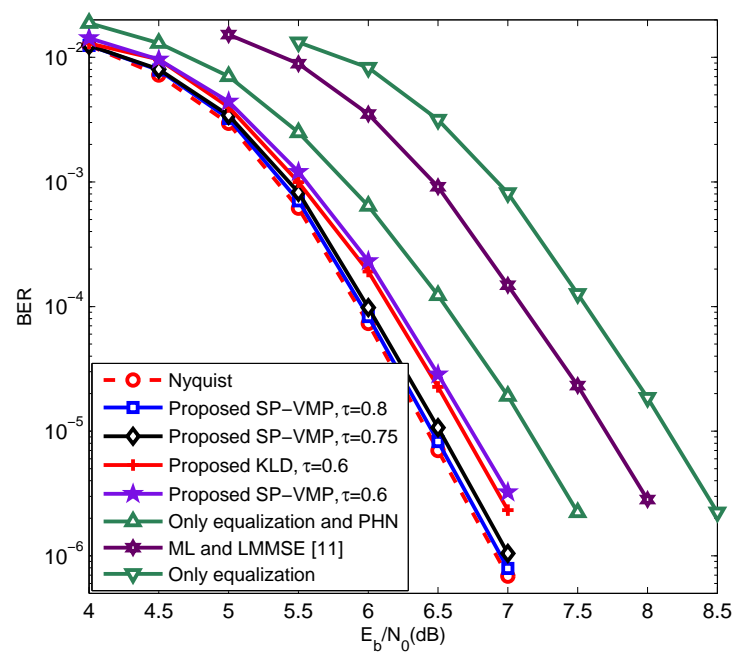

Figure 4: BER performance of the proposed algorithm and comparison with other algorithms with $\sigma_{\Delta}=0.1^{\circ}$. 


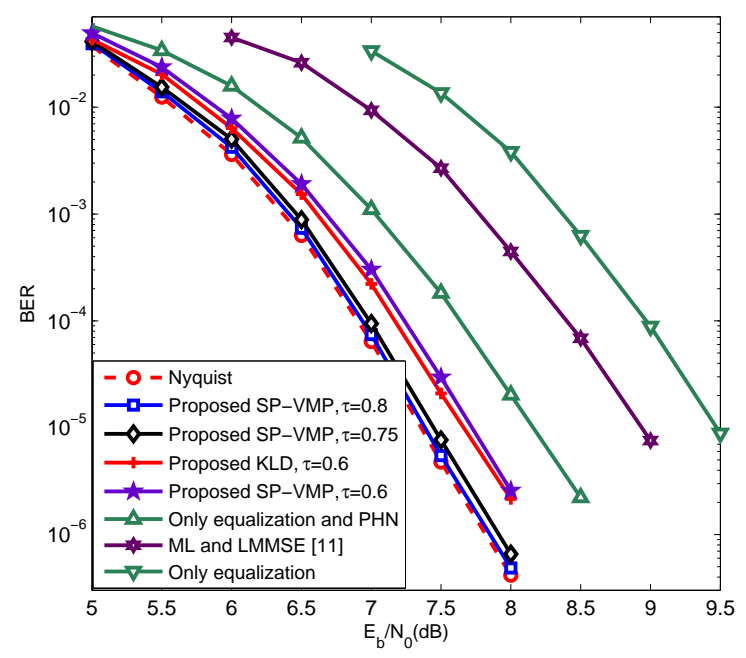

Figure 5: BER performance of the proposed algorithm and comparison with other algorithms with $\sigma_{\Delta}=3^{\circ}$.

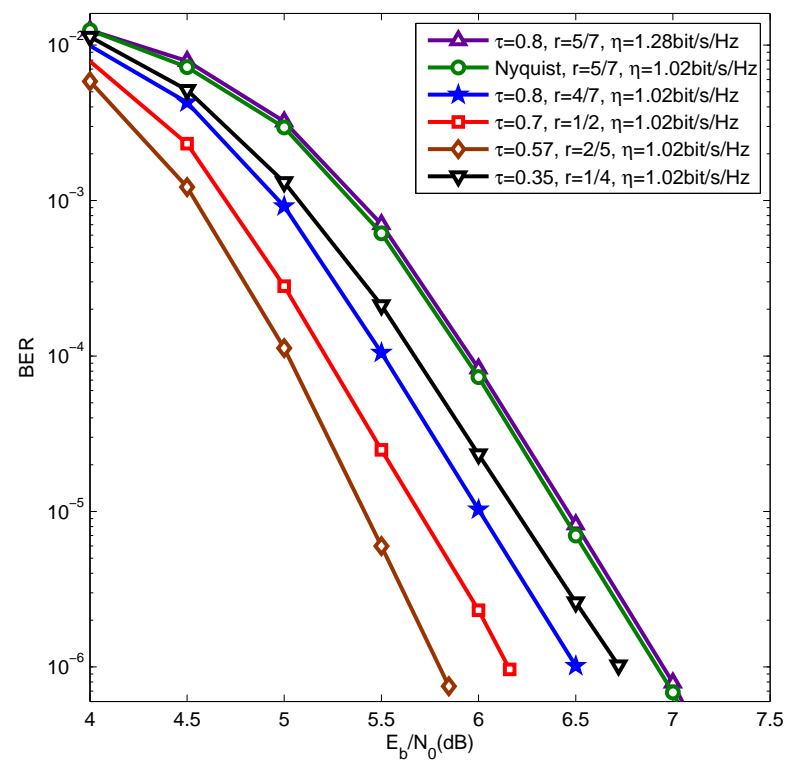

Figure 6: BER performance of the proposed algorithm with different $\tau$ and $r$. 


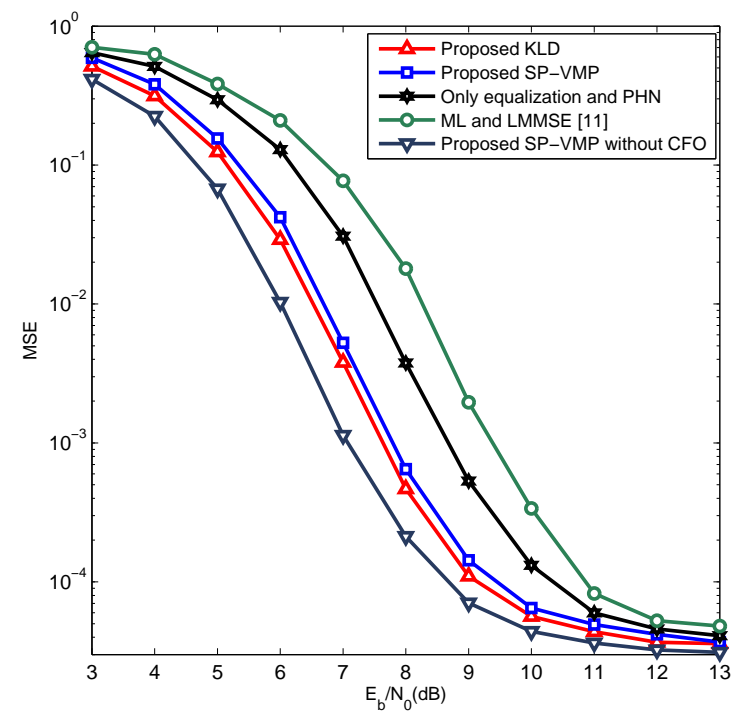

Figure 7: MSEs of the PHN estimation with $\sigma_{\Delta}=0.1^{\circ}$.

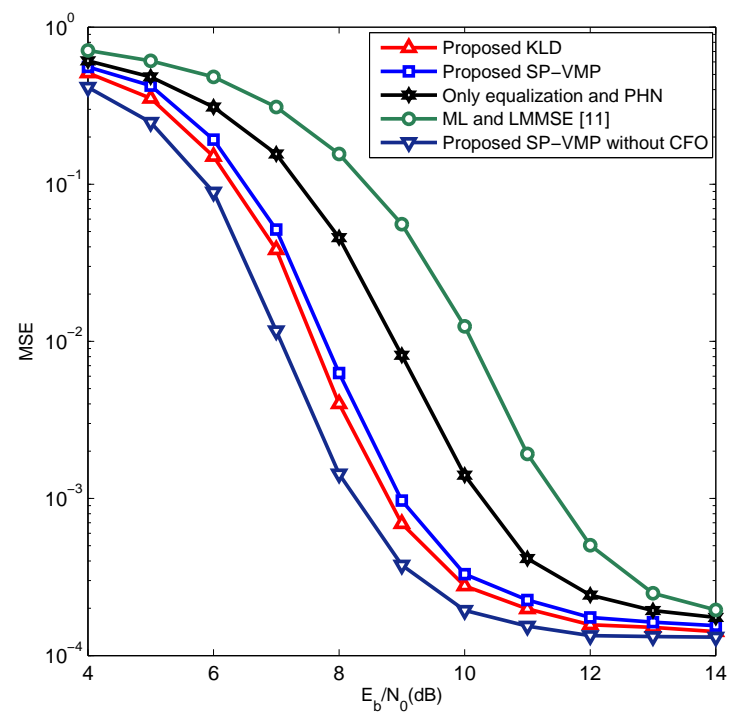

Figure 8: MSEs of the PHN estimation with $\sigma_{\Delta}=3^{\circ}$. 


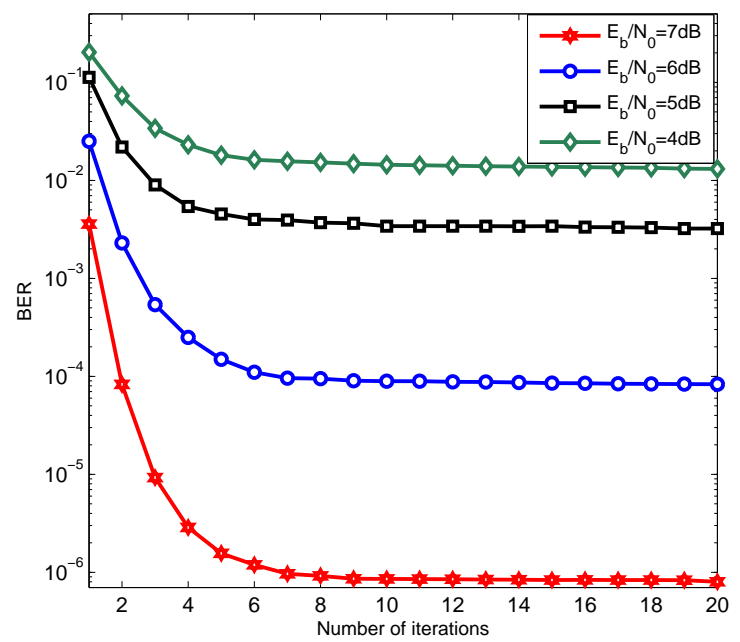

Figure 9: BER performance of the proposed algorithm with different iteration numbers.

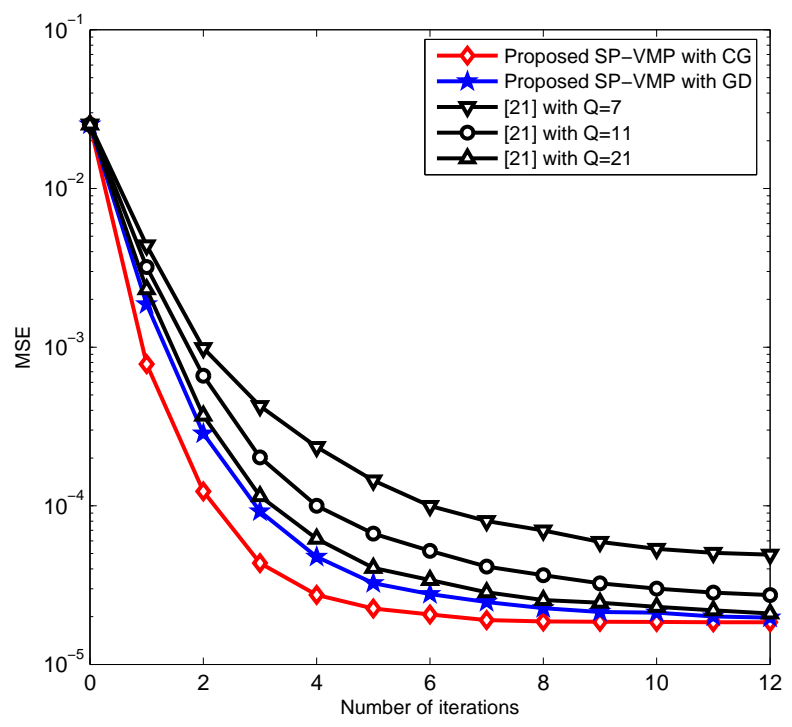

Figure 10: MSEs of the the proposed CFO estimation $\hat{\varphi}$ and comparison with different algorithms. 
$\overline{\text { Algorithm } 1 \text { Iterative Detection of FTN Signaling in the Presence of PHN }}$ and $\mathrm{CFO}$

1: Initialization:

2: $\quad \mathbf{m}_{\mathbf{x}}^{\downarrow}=0, \mathbf{v}_{\mathbf{x}}^{\downarrow}=\mathbf{I} ; a_{f, 0}=0$ and $a_{b, K-1}=0 ; p(\varphi)$ is uniform distribution; $\mathbf{m}_{b \mathbf{s}_{K-1}}=0, \mathbf{V}_{b \mathbf{s}_{K-1}}=\mathbf{I} ; \mathbf{m}_{f \mathbf{s}_{0}}=0, \mathbf{V}_{f \mathbf{s}_{0}}=\mathbf{I} ;$

3: for $i=1$ to $I$ do

4: $\quad$-Message update for PHN and CFO estimation

5: $\quad$ Update forward and backward parameters $a_{f, k}$ and $a_{b, k}$ by (22) and (23);

6: $\quad$ Perform MAP estimation of $\varphi$ using CG by (18);

7: $\quad$-Message update for FTN symbol detection

8: $\quad$ Update $p_{u}\left(c_{k}\right)$ via KLD minimization or SP-VMP method;

9: $\quad$ For KLD minimization: Evaluate $p_{u}\left(c_{k}\right)$ by (25) and (27);

10: $\quad$ For $S P-V M P$ : Update parameters $m_{c_{k}}^{\uparrow}$ and $v_{c_{k}}^{\uparrow}(33)$ and (34);

11: $\quad$ Update parameters $m_{\omega_{k}}^{\uparrow}$ and $v_{\omega_{k}}^{\uparrow}$ by (36) and (37);

12: $\quad$ Update forward and backward parameters $m_{f \omega_{k}}, v_{f \omega_{k}}, m_{b \omega_{k}}$ and $v_{b \omega_{k}}$ by (40)-(43);

13: $\quad$ Update parameters $m_{\omega_{k}}^{\downarrow}$ and $v_{\omega_{k}}^{\downarrow}$ by (45) and (46);

14: $\quad$ Update parameters $\mathbf{W}_{b \mathbf{s}_{k}}^{\prime \prime} m_{b \mathbf{s}_{k}}^{\prime \prime}$ and $\mathbf{W}_{b \mathbf{s}_{k}}^{\prime \prime}$ by (48) and (49);

15: $\quad$ Update parameters $\mathbf{m}_{f \mathbf{s}_{k-1}}^{\prime \prime}$ and $\mathbf{V}_{f_{\mathbf{s}_{k-1}}}^{\prime \prime}$ by (54) and (53);

16: $\quad$ Update forward and backward parameters $\mathbf{m}_{f \mathbf{s}_{k-1}}, \mathbf{V}_{f \mathbf{s}_{k-1}}, \mathbf{m}_{b \mathbf{s}_{k-1}}$ and $\mathbf{V}_{b \mathbf{s}_{k-1}}$ by (52), (51), (55) and (56);

17: $\quad$ Update parameters $m_{x_{k}}^{\uparrow}$ and $v_{x_{k}}^{\uparrow}$ by (58) and (59);

18: $\quad$ Update parameters of $p_{d}\left(c_{k}\right)$ by (61)-(64);

19: $\quad$-Message update for channel decoding

20: $\quad$ Update $p_{d}\left(x_{k}\right)$ by standard SPA decoding. 